\title{
Erratum: Equilibrium probability distribution of a conductive sphere's floating charge in a collisionless, drifting Maxwellian plasma [Phys. Rev. E 88, 023110 (2013)]
}

Drew M. Thomas and Michael Coppins

(Received 27 January 2014; published 10 February 2014)

DOI: 10.1103/PhysRevE.89.029903

PACS number(s): 52.27.Lw, 05.40.-a, 99.10.Cd

Equation (66) of the published paper has a typographical error: A forward slash is missing from a denominator. The equation should, therefore, read

$$
f_{N}=\sqrt{\frac{-y_{0}^{\prime}}{2 \pi}} \exp \left[-\frac{\left(N-N_{0}\right)^{2}}{2 /-y_{0}^{\prime}}\right] .
$$

Also, on the same page, the paragraph beginning with "While the dependence of $\sigma^{2}$ on $\alpha$ goes as $O\left(T_{e} a\right)$ " should begin, "While the dependence of $\sigma^{2}$ on $\alpha$ goes as $O(1 / \alpha)$ ".

These corrections alter neither the results nor the conclusions of the paper. 